\title{
SELBSTORGANISATION
}

\section{Wie Sie die Dinge geregelt kriegen}

Stephan List

Seinen Tag und seine Arbeit sinnvoll und flexibel zu strukturieren, gehört zur hohen Kunst des Managements. Der Computer kann dabei helfen.

Die meisten Menschen machen im Beruf die gleiche Erfahrung: Die Arbeit wird mehr, die Zeit zu ihrer Erledigung knapper. Wir ertrinken in Informationen, es fällt zunehmend schwer, die Spreu vom Weizen zu trennen. Umso wichtiger ist es, den Überblick zu bewahren und sich clever zu organisieren.

Für viele beginnt der Arbeitstag mit dem Anschalten des Personalcomputers und endet abends mit dem Herunterfahren. Er läuft den ganzen Tag, mal mehr, mal weniger klaglos. Warum den Computer nicht als Hilfe nutzen, zumal er Organisationsaufgaben gleichsam nebenbei erledigt?

Es gibt eine Reihe kleiner Programme, die das Arbeitsleben leichter machen und dazu kostenlos sind. Die folgende Übersicht zeigt, wo man sie zweckmäßig einsetzen kann.

\section{Notizen}

Ein Geburtstag, den viele verpasst haben: Der Haftzettel wurde unlängst 25 Jahre alt. Seitdem die »post-it-note «® erfunden wurde, kleben sie überall, die gelben, roten, grünen Zettel. Auf Bildschirmen und Schreibtischunterlagen sind sie ebenso zu finden, wie in Büchern als Lesezeichen. Zumindest den Bildschirm kann man zettelfrei halten, wenn man Klebezettel in elektronischer Form verwendet. Mit »Sticky Notes « oder »Jhoos Notes« lassen sich virtuelle Haftzettel an den Bildschirm kleben und beliebig hinund herschieben. Sogar in der Optik ähneln sie ihren Vorbildern. Mit »Jhoos Notes « können Sie Ihre Notizen sogar als E-Mail verschicken.

Für umfangreichere Notizen oder sogar Entwürfe, Berichte, Memos usw. reichen derlei Zettel nicht mehr aus. Ein Programm mit umfangreichen Features ist »TiddlyWiki «. In Anlehnung an das Wiki-Format, das auch bei der bekannten Wikipedia-Enzyklopädie verwendet wird, lassen sich umfangreiche Notizen erstellen, die man mit Links untereinander vernetzen kann. Jede Notiz kann mit Stichwörtern, so genannten Tags versehen werden, die das spätere Auffinden gewünschter Informationen einfach machen. Eine Journalfunktion ermöglicht das chronologische Einstellen von Texten, einem Arbeitstagebuch

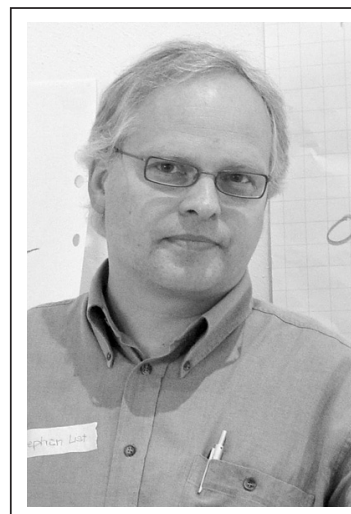

Dr. Stephan List ist freiberuflicher Trainer und Organisationsberater mit Schwerpunkt in der Industrie. Er ist Spezialist für Arbeitsmethodik und Arbeitsorganisation und berät auf diesen Gebieten Unternehmen und Einzelpersonen. Auf seiner Website »ToolBlog « gibt er regelmäßig Tipps zum Selbstmanagement. Dort finden Sie auch die Links zu den vorgestellten Programmen direkt zum Anklicken. Internet http://www.toolblog.de

steht nichts mehr im Wege. Der Clou: »TiddlyWiki « braucht nicht installiert zu werden, es läuft quasi als Internetseite. Man braucht lediglich einen herkömmlichen Internetbrowser, wie Firefox oder Internet Explorer.

\section{Strukturieren}

Sei es die Planung eines Projektes oder die Vorbereitung einer Präsentation: Für viele Aufgaben ist es erforderlich, vorhandene Informationen auf zweckmäßige Weise zu strukturieren und zu gliedern. Methode der Wahl ist für viele das »Mind-Mapping « nach Tony Buzan. Es eignet sich gleichwohl zur Ideenfindung als auch zur Strukturierung und Weiterverarbeitung von Informationen. Ein kostenloses Programm, mit dem man Mind-Maps auf dem Computer erstellen kann, ist »FreeMind «. Das Programm steht kommerziellen Konkurrenten keineswegs nach. Es findet sich alles, was für das Zeichnen von MindMaps notwendig und komfortabel ist.

»Concept Mapping « ist ein anderes Verfahren, Informationen zu visualisieren. Es ähnelt ein bisschen der Kartentechnik, die viele von Seminaren und Workshops kennen. Informationen werden auf Karten geschrieben, die Karten dann auf einer Pinnwand in logische Beziehungen zueinander gebracht. Mit »Cmap Tools« ersetzen sie die Pinnwand durch ihren Bildschirm. Sämtliche »Karten « können übrigens auch mit eigenen Notizen, Bildern usw. unterlegt werden. 


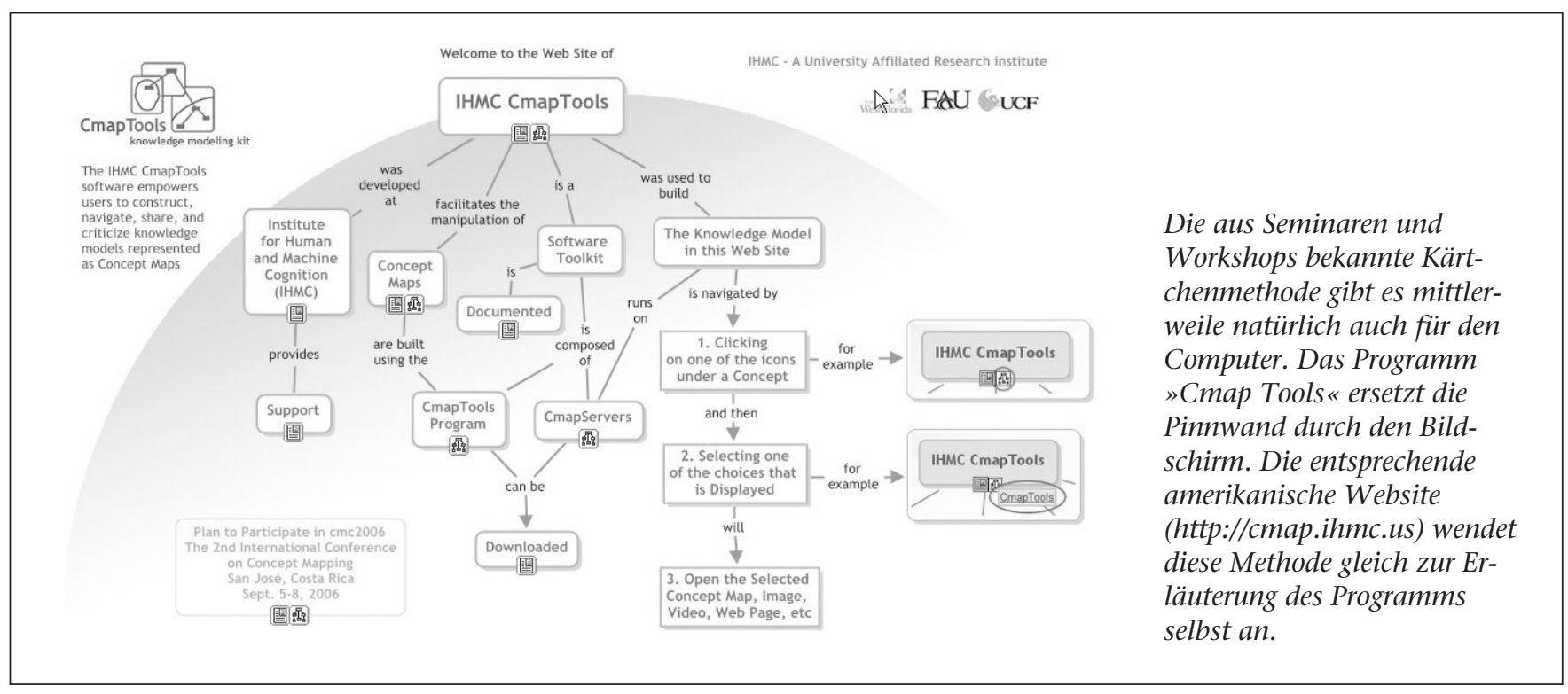

Wer die Schrift dem Bild vorzieht, für den ist »KeyNote « die richtige Wahl. Hier lassen sich die Notizeinträge hierarchisch in Form einer Gliederung darstellen. Verweise zu Internetseiten oder anderen Dateien sind, wie bei den anderen beiden Programmen, kein Problem.

\section{Zeitmanagement}

Die knappe Zeit so einteilen, dass die Aufgaben zur festgesetzten Zeit erledigt werden, ist das Kernthema des

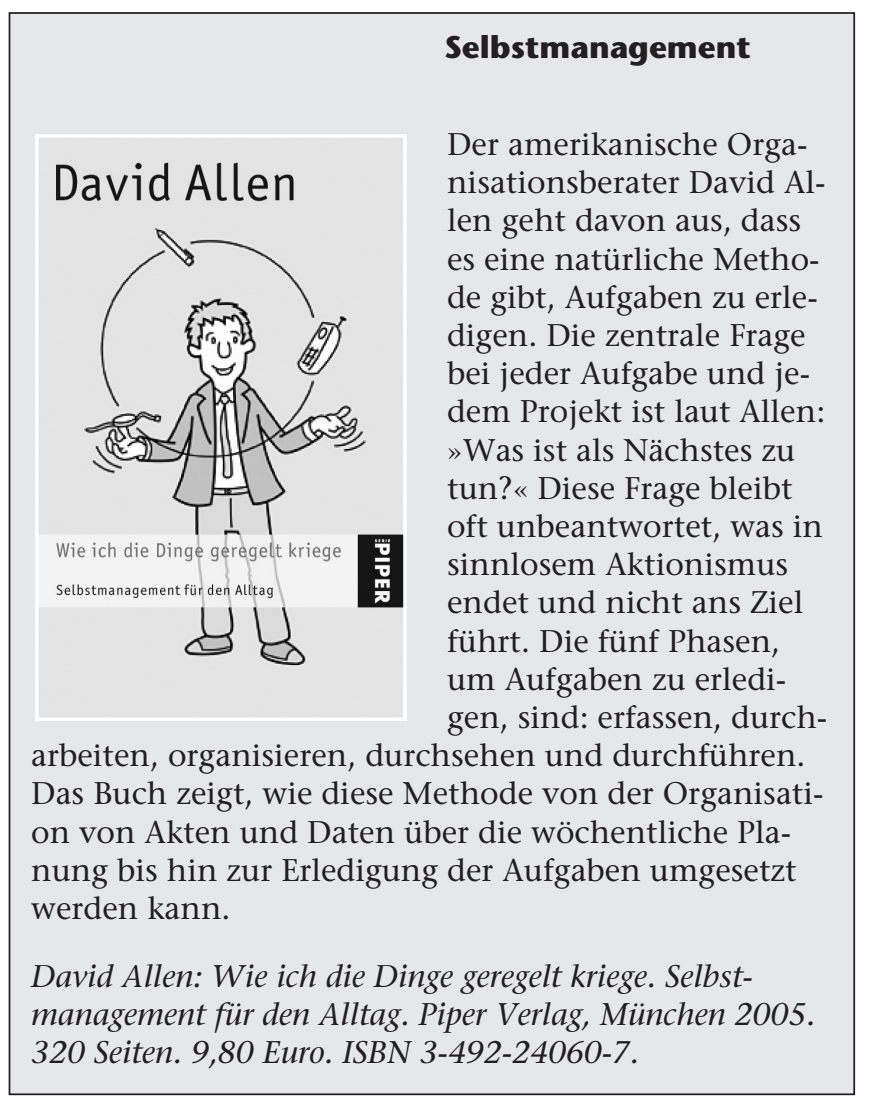

Zeitmanagements. Leider gibt es kein Programm, das es Ihnen abnimmt, Ziele zu definieren, Aufgaben zu beschreiben und Prioritäten zu setzen, wohl kann Sie aber Software dabei unterstützen.

Die Programme, die eine Alternative zu Microsoft Outlook darstellen wollen, ist mittlerweile unüberschaubar. Besonders interessant sind die Angebote im Internet. Die Vorteile: Man muss nichts auf dem lokalen Rechner installieren und die eigenen Daten sind nach Eingabe von Benutzernamen und Passwort von jedem Rechner aus erreichbar, der Zugang zum Internet hat. Der Nachteil: Man muss den Anbietern hinsichtlich der Privatsphäre vertrauen und meist etwas Werbung ertragen.

Ein typischer Vertreter dieser Gattung ist das persönliche Portal des Suchmaschinenbetreibers »yahoo!«. Es heißt einfach »mein yahoo! « und stellt nach dem Registrieren alles zur Verfügung, was man für die persönliche Arbeitsorganisation braucht: Kalender, Aufgabenliste, sogar ein E-Mail-Konto. Man kann seine Internetlesezeichen (Bookmarks) genauso ablegen, wie sich Wetterdaten anzeigen lassen. Die Inhalte können beliebig nach eigenem Gusto zusammengestellt werden. Die Flexibilität dieses Dienstes hat allerdings ihren Preis: Sehr schnell wird es unübersichtlich und auch die Bedienung ist nicht immer einfach.

Wem es da zu kompliziert wird, kann auf Online-Kalender wie »HipCal« oder »CalendarHub « ausweichen, die eine Fülle von nützlichen Merkmalen bieten wie gemeinsame Nutzung mit Kollegen oder das Erinnern an Termine über E-Mail. Bei »CalenderHub« können Sie sogar Ihre Daten aus Outlook importieren und weiterbearbeiten.

Eine einfache, aber dennoch wirkungsvolle Art, seine Arbeit zu organisieren, ist das Erstellen von Aufgabenlisten. Sie finden ihr elektronisches Gegenstück in Angeboten wie »voo2do« oder »e2do«. Deren Namen sind Programm, handelt es sich doch um die Verballhornung des englischen Begriffes »to-do-list «. Hier trägt man einfach seine Aufgaben mit Start- und Enddatum ein und hakt sie $a b$, wenn sie erledigt sind. 


\section{Zeitmanagement einmal anders: Getting Things Done (GTD)}

Ziele und Aufgaben definieren, Prioritäten festlegen, die Dauer der Aufgaben abschätzen und den Tagesablauf entsprechend planen, war die Vorgehensweise des Zeitmanagements, wie es üblicherweise in den vielen einschlägigen Seminaren gelehrt wird. Indes ließ die Umsetzung dieses Ansatzes in die Praxis meist zu wünschen übrig.

Vor einigen Jahren erschien in den USA ein Buch mit dem Titel »Getting Things Done« (vgl. Seite 19), in dem der Autor David Allen einen völlig anderen Ansatz beschrieb. Mittlerweile ist »GTD« ein eigener Begriff geworden, die Methode hat eine aktive Anhängerschaft, die sich lebhaft in Foren auf Internetseiten und in Maillisten austauscht.

Für Allen sind Prioritäten eher zweitrangig, er kategorisiert Aufgaben vielmehr danach, wo man sie erledigen kann und wie viel Zeit oder Energie man dafür hat. So gibt es eben Aufgaben, die ich nur im Büro am PC erledigen kann, während andere auch während einer halbstündigen Straßenbahnfahrt getan werden können. Alles in allem ist »GTD « eine andere Philosophie des Selbstmanagements. »GTD« ist nicht nur einleuchtend, sondern funktioniert auch in der Praxis.

Natürlich gibt es mittlerweile auch Programme, die den Ansatz von »GTD« unterstützen. »GTD TiddlyWiki« und

\section{Wo Sie die erwähnten Programme finden}

\section{Notizen}

Sticky Notes: http://www.sticky-notes.net

Jhoos Notes: http://notes.jhoos.com

TiddlyWiki: http://www.tiddlywiki.com

\section{Strukturieren}

FreeMind: http://freemind.sourceforge.net

Cmap Tools: http://cmap.ihmc.us

KeyNote: http://www.tranglos.com/free/keynote.html

\section{Zeitmanagement}

HipCal: http://www.hipcal.com

CalendarHub: http://www.calendarhub.com

voo2do: http://voo2do.com

e2do: http://www.e2do.com

\section{Zeitmanagement einmal anders: Getting Things Done (GTD)}

GTD TiddlyWiki: http://shared.snapgrid.com/gtd_tiddlywiki.html

NextAction: http://trimpath.com/project/wiki/NextAction

\section{Gemeinsam arbeiten}

FreePDF XP: http://freepdfxp.de/freepdf.htm

writely: http://www.writely.com

iRows: http://irows.com/

Tumbstacks: http://thumbstacks.com/

\section{Ausblick}

del.icio.us: http://del.icio.us
»NextAction« sind zwei Vertreter dieser Software. Beide benötigen zur Ausführung lediglich einen Internetbrowser. Die Bedienung der Programme setzt allerdings ein Grundverständnis von »GTD « voraus. Sie sollten die deutsche Übersetzung von Allens »Getting Things Done« gelesen haben, wenn Sie die Programme einsetzen wollen. Die Lektüre des Buches lohnt sich aber allemal, »GTD « beschränkt sich nicht nur auf die Organisation der täglichen Arbeit, sondern gibt Hinweise zur Dokumentenablage, zur Planung von Projekten und vielem mehr.

\section{Gemeinsam arbeiten}

Das gemeinsame Arbeiten an Dokumenten erfolgte bislang vor allem durch das Hin-und Herschicken von E-Mails, an die man die entsprechenden Dokumente als Dateien anhängte. Das funktioniert nicht immer reibungslos, zum einen benutzen die Partner nicht immer dasselbe Dateiformat, zum anderen ist bei vielen Firmen bei Anhängen die Dateigröße nach oben begrenzt, was das Versenden umfangreicher Dokumente unmöglich macht.

Das Problem des unterschiedlichen Dateiformats lässt sich lösen, indem man das Dokument in eine PDF-Datei umwandelt, die mit dem frei erhältlichen »Acrobat Reader« gelesen werden kann. Dieser befindet sich auf nahezu jedem Bürorechner. Eine PDF-Datei können Sie mit der Freeware »FreePDF XP« erzeugen. Das Programm funktioniert im Prinzip wie ein weiterer Drucker. Wählt man als »Drucker« das Programm »FreePDF XP«, wird das Dokument nicht zum angeschlossenen Drucker geschickt, sondern vielmehr eine PDF-Datei erzeugt, die man anschließend an beliebiger Stelle abspeichern kann. Allerdings kann Ihr Partner das Dokument zwar lesen, aber in der Regel nicht weiterverarbeiten.

Diese Lücke schließen Internetdienste wie »writely«. Nach der üblichen Registrierung können Sie neue Dokumente erstellen oder Ihre Texte im Word-, Rich Text- oder OpenOffice-Format hochladen und weiter bearbeiten, es ist keine zusätzliche Software nötig, Ihr Browser genügt. Die Bedienung ist genauso schnell und einfach wie bei Ihrer Textverarbeitung, eine neue Technik namens »Ajax « macht dies möglich. Per E-Mail können Sie Partnern und Kollegen die Freigabe erteilen, das Dokument entweder anzusehen oder sogar zu überarbeiten.

Was »writely« für Textverarbeitung, ist »iRows« für Tabellenkalkulation. Auch hier können Sie Tabellenrechnungen online erstellen oder hochladen und weiter bearbeiten.

Damit das Online-Büropaket komplett wird, fehlt nur noch die geeignete Software zum Erstellen von Präsentationen, das Gegenstück zu »PowerPoint«. Und auch hier werden Sie im Internet fündig. »Tumbstacks« heißt das Angebot. Anschauen kann man die fertige Präsentation entweder online im Webbrowser oder als separate HtmlDatei offline.

»Writely«, »iRows « und »Thumbstack« setzen auf neueste Programmiertechnologie und sind dadurch sehr einfach zu bedienen. Allerdings befinden sie sich auch teil- 
weise im Entwicklungsstatus, sodass nicht immer alle Merkmale freigegeben sind oder reibungslos funktionieren. Dennoch erscheinen sie durchaus jetzt schon praxistauglich.

\section{Ausblick}

Gerade die zuletzt angeführten Programme lassen erahnen, dass das Internet ganz neue Möglichkeiten in der Organisation der Arbeit eröffnet.

Von der Verwendung neuer Techniken war schon die Rede. Ein anderer Ansatz ist die Vernetzung der Nutzer über das so genannte »Tagging «. Tags sind Etiketten oder Schlagwörter, mit denen die Internetnutzer ihre Dateien beim Abspeichern bei den verschiedenen Diensten versehen. Diese Dateien können Bilder sein, Texte oder auch Bookmarks genannte Lesezeichen, mit denen man sich interessante Websites »merkt«. Einer dieser BookmarkDienste ist »del.icio.us «. Habe ich beispielsweise eine interessante Seite zu Kuchenrezepten gefunden, versehe ich die Seite mit dem Tag »Rezepte«. Wenn ich die Seite später wieder aufsuchen will, gebe ich bei »del.icio.us « ins Suchfeld »Rezepte« ein. Als Ergebnis erhalte ich nicht nur die Seiten, die ich unter »Rezepte« eingegeben habe, sondern getrennt davon auch die der anderen del.icio.usNutzer unter diesem Stichwort. Dies ist ein idealer Ausgangspunkt für die Recherche, was es sonst noch für Rezeptseiten gibt.

Viele Experten halten derartige Entwicklungen für so bahnbrechend, dass sie bereits heute von »web 2.0 « sprechen, dem Internet in der neuen Version.

Man darf gespannt sein, welche Werkzeuge das Netz in Zukunft für Arbeitsorganisation und Informationsverarbeitung zur Verfügung stellt. An uns liegt es, sie sinnvoll zu nutzen.

\section{Wie ich mich organisiere}

Interessant ist es immer zu erfahren, wie individuell jeder Einzelne die Methode von Getting Things Done (GTD) umsetzt. Deshalb hier, wie ich mich organisiere.

\section{Listen}

Ich führe die Listen als einfache Text-Dokumente im txt-Format. Meine Listen sind:

- Projekte.txt, unterteilt in
O ARBEIT
PRIVAT
PERSÖNLICH

- Next_Actions.txt, unterteilt in

$$
\text { ○ IM BÜRO }
$$

o ZU HAUSE

- Vielleicht-Irgendwann.txt, unterteilt in

O BÜRO

O PRIVAT

O FERIEN/REISEN

- Warten_auf.txt, unterteilt in

$$
\text { O BÜRO }
$$

O PRIVAT

\section{E-Mail}

In meinen E-Mail-Programmen (zu Hause und am Arbeitsplatz identisch) habe ich Ordner angelegt, in die ich E-Mails aus dem Posteingang sofort einsortiere. Die Ordner sind:

- ^Als nächstes tun (»Next Actions«)

- ^Leute kontaktieren (»Agenda «)

- ^Projekte, je Projekt ein Unterordner

- ^Vielleicht später (»Someday/Maybe«)

- ${ }^{\wedge}$ Warten auf (»Waiting For «)

David Allen empfiehlt, ein grafisches Zeichen dem Ordnernamen voranzustellen. Bei mir ist es das ^^-Zeichen. Es hilft beim schnellen Finden der Ordner und macht, dass sie in der automatischen Sortierung zuoberst erscheinen.

\section{Kalender}

$\mathrm{Zu}$ Hause benutze ich »iCal« für Termine und zur Erinnerung an Aktionen an einem bestimmten Tag. In der Arbeit benutze ich den Kalender unserer Groupware für die gleichen Aufgaben.

\section{Webserver}

Auf meiner Website habe ich einen Bereich eingerichtet, auf den ich von der Arbeit aus am Ende des Tages die Listen via FTP ablege. Zu Hause bearbeite ich die Listen auf dem Webserver.

\section{Mobiltelefon}

Den Kalender zu Hause kann ich mit meinem SonyEricsson K7501 synchronisieren. Mit aufs Handy kommt auch die Liste mit den »Aufgaben« zur Erinnerungen an zeitlich bestimmte Aktionen. Via mobilen Internetzugang kann ich auf die Listen auf meinem Webserver zugreifen. Ab und zu erfasse ich auch eine Notiz oder einen Termin über die Handytastatur.

\section{Notizblock}

Listen führe ich darauf keine. Ich nutze ihn bei Bedarf als »Eingang «, in dem ich mir Notizen mache.

\section{Ablage}

Bei der Arbeit bewahre ich Materialien für Projekte in Plastik-Sichtmäppchen je Projekt auf (»Project Support Materials«). Zum Anschreiben nehme ich Adress-Klebettiketten und beschrifte sie mit Bleistift. Eine umfassendere Ablage mit Hängemappen stelle ich erst zusammen. $\mathrm{Zu}$ Hause habe ich eine alphabetische Ablage mit Hängemappen. Die aber ist voll gestopft, sodass ich nichts mehr ablegen kann; genau das Problem, wie David Allen es beschreibt. Stefan Bucher

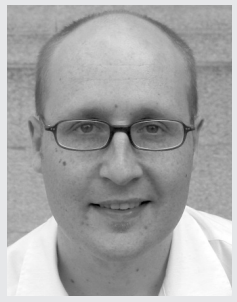

Stefan Bucher aus Zürich arbeitet als Publishing Specialist im Bereich Customer Care bei einem Telekommunikationsanbieter. Seit Anfang dieses Jahres organisiert er sich nach der Methode »Getting Things Done " von David Allen. Seine Website: http://www.stefanbucher.net. 